\title{
Quantum phases in the frustrated Heisenberg model on the bilayer honeycomb lattice
}

\author{
Hao Zhang, ${ }^{1, \text { f }}$ M. Arlego, ${ }^{2}$ and C. A. Lamas, ${ }^{2}$, \\ ${ }^{1}$ Institute for Solid State Physics, University of Tokyo, Kashiwa, Chiba 277-8581, Japan \\ ${ }^{2}$ IFLP - CONICET, Departamento de Física, Universidad Nacional de La Plata, C.C. 67, 1900 La Plata, Argentina.
}

\begin{abstract}
We use a combination of analytical and numerical techniques to study the phase diagram of the frustrated Heisenberg model on the bilayer honeycomb lattice. Using the Schwinger boson description of the spin operators followed by a mean field decoupling, the magnetic phase diagram is studied as a function of the frustration coupling $J_{2}$ and the interlayer coupling $J_{\perp}$.

The presence of both magnetically ordered and disordered phases is investigated by means of the evaluation of ground-state energy, spin gap, local magnetization and spin-spin correlations. We observe a phase with a spin gap and short range Néel correlations that survives for non-zero next-nearest-neighbor interaction and interlayer coupling. Furthermore, we detect signatures of a reentrant behavior in the melting of Néel phase and symmetry restoring when the system undergoes a transition from an on-layer nematic valence bond crystal phase to an interlayer valence bond crystal phase. We complement our work with exact diagonalization on small clusters and dimerseries expansion calculations, together with a linear spin wave approach to study the phase diagram as a function of the spin $S$, the frustration and the interlayer couplings.
\end{abstract}

PACS numbers: 75.10.Jm, 75.50.Ee, 75.10.Kt

\section{INTRODUCTION}

The study of the possible disordered ground states on the honeycomb lattice has received a great interest in the last years. The interest is focused mainly on the existence of quantum spin liquids in quantum antiferromagnets $1-\underline{-5}$. Recently, possible quantum disordered phases have been reported in the phase diagram corresponding to the single layer honeycomb Heisenberg mode $\underline{1}^{6-23}$. From the theoretical point of view, it is interesting to study the influence of an interlayer coupling in the stabilization of these disordered phases. In particular in the bilayer models, the ground state corresponding to very large values of the interlayer couplings should be a dimer product state. For unfrustrated models a transition between the Néel phase and the dimer phase is expected to obtain as the interlayer coupling is increased. This "melting" of Néel order can be studied as a function of the frustration in each layer. By contrast in the frustrated case, the system might go from a nonmagnetic nematic phase to a dimer product state as the interlayer coupling is increased.

From the experimental side, a very exciting progress on the bismuth oxynitrate, $\mathrm{Bi}_{3} \mathrm{Mn}_{4} \mathrm{O}_{12}\left(\mathrm{NO}_{3}\right)$, was obtained by Smirnova et al $\stackrel{24}{\underline{s}}$. The magnetic susceptibility data indicate two-dimensional magnetism. Despite the large AF Weiss constant of - $257 \mathrm{~K}$, no long-range ordering was observed down to $0.4 \mathrm{~K}$, which suggests a nonmagnetic ground state $\underline{24-27}$. In this compound the $\mathrm{Mn}^{4+}$ ions form a honeycomb lattice without any distortion. Two layers of such honeycomb lattices are separated by bismuth atoms, forming a bilayer structure with these bilayers separated by a large distance. Thus, the appropriate geometry to describe its magnetic properties is the bilayer honeycomb lattice. The magnetic exchange coupling constants have been calculated using a density functional theory, which shows that the dominant interactions are the intra-layer nearest-neighbor interaction

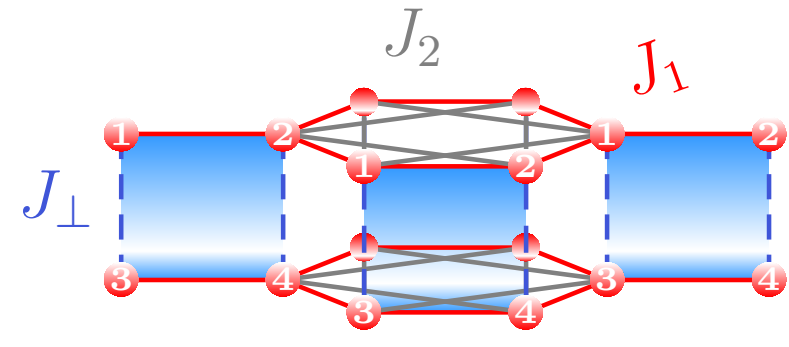

FIG. 1. (Color online) Schematic representation of the relevant couplings interactions in $\mathrm{Bi}_{3} \mathrm{Mn}_{4} \mathrm{O}_{12}\left(\mathrm{NO}_{3}\right)$. Colored areas correspond to the unit cells. The sites in each unit cell are labeled from 1 to 4 .

$J_{1}$ and the effective interlayer interaction $J_{\perp} \underline{28}$.

In Ref. 26, Matsuda et. al. have found experimental evidence that $J_{1}, J_{2}$ and $J_{\perp}$ are the dominant couplings and there is competition between them. As a result of this competition, a disordered ground state has been found. This observation has raised the interest in the study of magnetically disordered phases in honeycomb lattice antiferromagnets. Most of the advances have been reached in the single layer honeycomb lattice $\underline{-22,29,30}$ whereas less attention has been given to the unfrustrated bilayer case $\mathrm{e}^{30}-32$. Therefore, there are still many open issues, especially for the frustrated bilayer case.

The aim of this paper is to study the zero temperature phase diagram of the frustrated Heisenberg model on the bilayer honeycomb lattice. The dependence on the interlayer coupling $J_{\perp}$ is investigated for different values of the frustration $J_{2}$. We focus on the $S=1 / 2$ case, where quantum fluctuations become more important. The present study has several motivations. On the one hand, the phase diagram corresponding to $S=1 / 2$ is a natural 
extension of the recently presented phase diagram for the single layer honeycomb lattice ${ }^{9}$. On the other hand, the substitution of $\mathrm{Mn}^{4+}$ in $\mathrm{Bi}_{3} \mathrm{Mn}_{4} \mathrm{O}_{12}\left(\mathrm{NO}_{3}\right)$ by $\mathrm{V}^{4+}$ may lead to the realization of the $S=1 / 2$ Heisenberg model on the honeycomb lattice.

In this paper, using the Schwinger boson representation followed by a mean field decoupling, the presence of both magnetically ordered and disordered phases is investigated. We observe signatures of a reentrant behavior in the melting of Néel phase. The behavior of the local magnetization as a function of the interlayer coupling $J_{\perp}$ gives a physical explanation to this effect, since a small $J_{\perp}$ makes the system more magnetically ordered. Another key finding of our work is that the interlayer coupling may restore the lattice rotational symmetry within layers. Furthermore, the linear spin wave theory (LSWT) is used to describe the general behavior as a function of the spin $S$. To support the mean-field results Lanczos technique in small systems is used, complemented with series expansion based on the continuous unitary transformation method to estimate the ground-state energy and the triplet gap. Last but not least, a comparison with previous Schwinger boson mean-field results for the $S=1 / 2$ Heisenberg mode $\underline{\underline{9}}^{\underline{n}}$ on the single-layer case is discussed.

The outline of the paper is as follows: In Sec. II we introduce the model. In Sec. III we apply the Schwinger boson mean-field approach for $S=1 / 2$ case, complemented with Lanczos technique. In Sec. IV we apply LSWT for general spin $S$. A comparison of the groundstate energy and the triplet gap obtained by means of series expansion and Lanczos technique is presented in Sec. V. We close with a discussion and conclusions in Sec. VI

\section{FRUSTRATED BILAYER HEISENBERG MODEL}

The Heisenberg model on the bilayer honeycomb lattice is described by

$$
H=\sum_{\vec{r}, \vec{r}^{\prime}, \alpha, \beta} J_{\alpha, \beta}\left(\vec{r}, \vec{r}^{\prime}\right) \overrightarrow{\mathbf{S}}_{\alpha}(\vec{r}) \cdot \overrightarrow{\mathbf{S}}_{\beta}\left(\vec{r}^{\prime}\right)
$$

where, $\overrightarrow{\mathbf{S}}_{\alpha}(\vec{r})$ is the spin operator on site $\alpha$ corresponding to the unit cell $\vec{r}$. $\alpha$ takes the values $\alpha=1,2,3,4$ corresponding to the four sites on each unit cell as depicted in Fig. 1. The coupling constants $J_{\alpha, \beta}\left(\vec{r}, \vec{r}^{\prime}\right)$ on the bonds of the bilayer lattice are depicted in Figure 1

The classical model displays Néel order for $J_{2} / J_{1}<$ $1 / 6$. The interlayer coupling $J_{\perp}$ does not introduce frustration in the system and then, at the classical level and $T=0$, does not affect the classical Néel phase. In the quantum case the situation is much subtle, Néel order is likely to melt giving rise to a non-magnetic phase. In the following, except being explicitly specified, we fix the energy scale by taking $J_{1}=1$ to simplify the notation. For large values of $J_{\perp}$ we expect the ground state to be an interlayer valence bond crystal (IVBC) with corresponding spins from both layers forming dimers. There are two different ways to destroy the Néel order, by increasing the frustration on each layer or increasing the coupling between layers. The destruction of the Néel order in a single layer honeycomb lattice due to the frustration introduced by means of the next-nearest neighbors interactions has been studied by various approaches, including spin wave theory $\underline{10,30,33.34}$, a non-linear $\sigma$ model approach ${ }^{35}$, Schwinger boson mean-field theory (SBMFT) $)^{6,9}, \underline{48}$, bond operator mean-field theory $\underline{\underline{10}}$, exact diagonalization $(\mathrm{ED})^{11,12,34}$, a variational Monte Carlo (VMC) method ${ }^{18,19}$, series expansion $(\mathrm{SE})^{36}$, the pseudofermion functional renormalization group (PFFRG) $\underline{13}$, the coupled cluster method $(\mathrm{CCM}) \stackrel{15-17}{\underline{-17}}$ and the density matrix renormalization group (DMRG) $\operatorname{method}^{20}-22$.

For the single layer case the most accepted scenario is that at a critical value of the frustrating coupling $J_{2}$ the Néel order is destroyed giving rise to a magnetically disordered phase. The different techniques listed above have yielded strong evidence supporting the existence of an intermediate magnetically disordered region where a spin gap opens and spin-spin correlations decay exponentially $9.12,15,16,19$. This disordered region comprises two kinds of magnetically disordered phases distinguished by a rotational symmetry breaking. 9 . In the range $0.2075 \lesssim J_{2} / J_{1} \lesssim 0.3732$ there is a gaped spin liquid (GSL) phase, where the ground state is magnetically disordered and preserves all the lattice symmetries $\stackrel{9}{\text {. }}$.

For larger values of $J_{2}$ the system presents a ground state that breaks the lattice rotational symmetry, but preserves lattice translational symmetry. This staggered dimer valence-bond crystal ( $\mathrm{VBC}$ ), which is also called lattice nematic $\underline{10}$ was found by using a variety of techniques $9-12,18,20,21$. Finally for $J_{2} / J_{1} \gtrsim 0.398$, the system enters into a spiral phase $\mathrm{g}^{9.10}$. These phases (except the spiral one) are depicted in Figure 3-II.

Since the nematic phase present in the single layer case breaks the discrete rotational symmetry of the lattice, it is expected that, in the bilayer case, by increasing the interlayer coupling the system should undergo a transition from the nematic VBC to the IVBC phase, restoring the $Z_{3}$ symmetry.

As shown in the following sections, we study the Hamiltonian (1) using a rotationally invariant decomposition for the mean field parameters corresponding to a Schwinger boson representation of the spin operators, which has proven to be successful in incorporating quantum fluctuations $-\underline{7-9}, 37-44$. We complement this approach with ED, LSWT and SE. 


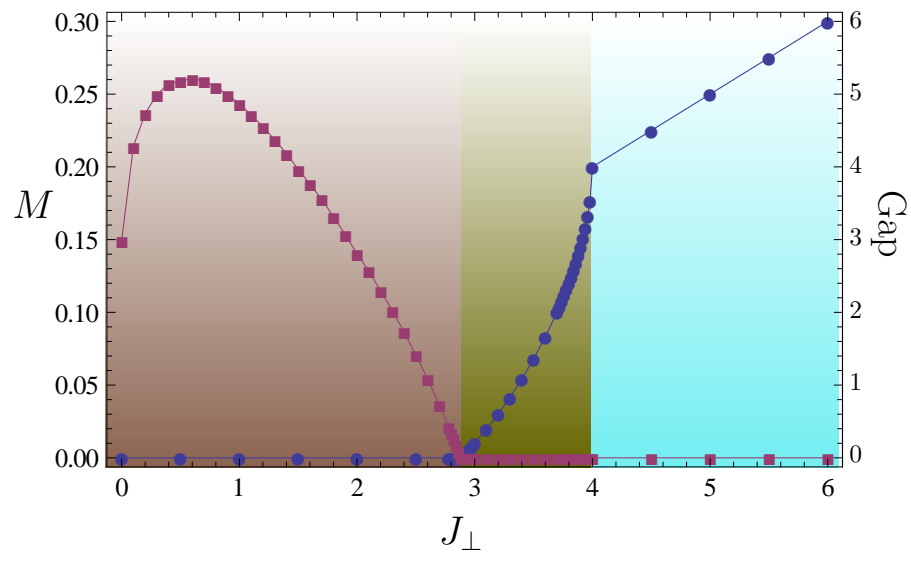

FIG. 2. (Color online) The spin gap (blue circles) and sublattice magnetization (red squares) obtained by SBMFT extrapolated to the thermodynamic limit, corresponding to the dashed line in Fig. $3\left(J_{2}=0.1\right)$. For $J_{\perp}>4$ the gap is proportional to $J_{\perp}$. Sublattice magnetization shows that Néel order is enhanced by small interlayer coupling, reaching a maximum at $J_{\perp} \sim 1 / 2$, after that it decreases until disappearing at $J_{\perp} \sim 2.9$. The brown shaded region corresponds to the Néel phase. In the green and light-blue regions, there is no evidence of any kind of magnetic order, and the light-blue region presents a gap that depends linearly with $J_{\perp}$.

\section{SCHWINGER BOSON MEAN-FIELD APPROACH AND EXACT DIAGONALIZATION}

In the Schwinger-boson representation, the Heisenberg interaction can be written as a biquadratic form. The spin operators are replaced by two species of bosons via the relation $\underline{45-\underline{47}}$

$$
\overrightarrow{\mathbf{S}}_{\alpha}(\vec{r})=\frac{1}{2} \overrightarrow{\mathbf{b}}_{\alpha}^{\dagger}(\vec{r}) \cdot \vec{\sigma} \cdot \overrightarrow{\mathbf{b}}_{\alpha}(\vec{r})
$$

where $\overrightarrow{\mathbf{b}}_{\alpha}(\vec{r})^{\dagger}=\left(\mathbf{b}_{\alpha, \uparrow}^{\dagger}(\vec{r}), \mathbf{b}_{\alpha, \downarrow}^{\dagger}(\vec{r})\right)$ is a bosonic spinor corresponding to the site $\alpha$ in the unit cell sitting at $\vec{r} . \vec{\sigma}$ is the vector of Pauli matrices, and there is a boson-number restriction $\sum_{\sigma} \mathbf{b}_{\alpha, \sigma}^{\dagger}(\vec{r}) \mathbf{b}_{\alpha, \sigma}(\vec{r})=2 S$ on each site.

In terms of boson operators we define the $S U(2)$ invariants

$$
\begin{aligned}
& \mathbf{A}_{\alpha \beta}(\vec{x}, \vec{y})=\frac{1}{2} \sum_{\sigma} \sigma \mathbf{b}_{\alpha, \sigma}(\vec{x}) \mathbf{b}_{\beta,-\sigma}(\vec{y}) \\
& \mathbf{B}_{\alpha \beta}(\vec{x}, \vec{y})=\frac{1}{2} \sum_{\sigma} \mathbf{b}_{\alpha, \sigma}^{\dagger}(\vec{x}) \mathbf{b}_{\beta, \sigma}(\vec{y}) .
\end{aligned}
$$

The operator $\mathbf{A}_{\alpha \beta}(\vec{x}, \vec{y})$ creates a spin singlet pair between sites $\alpha$ and $\beta$ corresponding to unit cells located at $\vec{x}$ and $\vec{y}$ respectively. The operator $\mathbf{B}_{\alpha \beta}(\vec{x}, \vec{y})$ creates a ferromagnetic bond, which implies the intersite coherent hopping of the Schwinger bosons.

In this representation, the rotational invariant spinspin interaction can be written as

$$
\overrightarrow{\mathbf{S}}_{\alpha}(\vec{x}) \cdot \overrightarrow{\mathbf{S}}_{\beta}(\vec{y})=: \mathbf{B}_{\alpha \beta}^{\dagger}(\vec{x}, \vec{y}) \mathbf{B}_{\alpha \beta}(\vec{x}, \vec{y}):-\mathbf{A}_{\alpha \beta}^{\dagger}(\vec{x}, \vec{y}) \mathbf{A}_{\alpha \beta}(\vec{x}, \vec{y})
$$

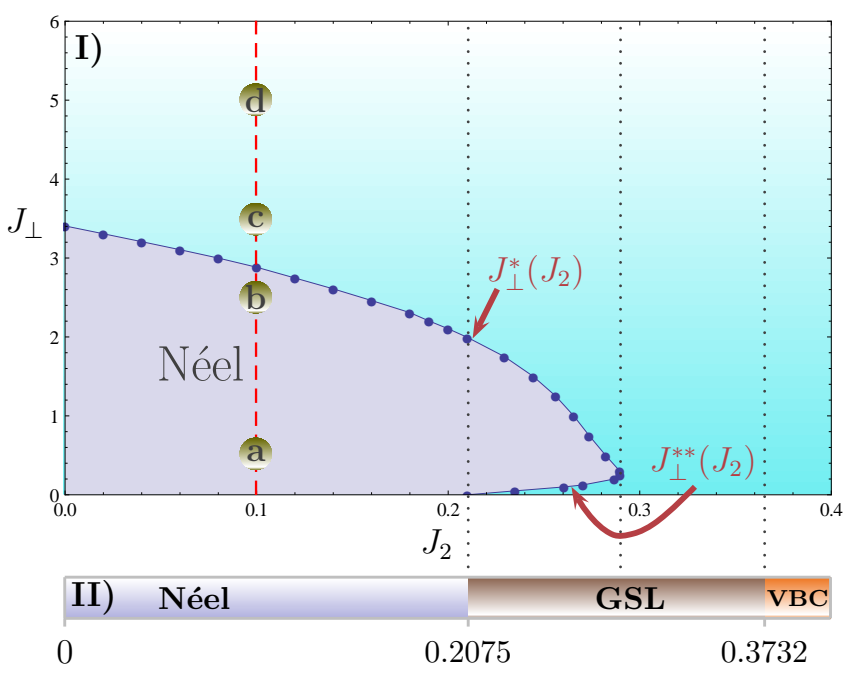

FIG. 3. (Color online) I) Phase diagram for $S=1 / 2$ in the $J_{2}-J_{\perp}$ plane obtained by means of SBMFT. Gray region correspond to the Néel phase whereas light-blue region corresponds to magnetically disordered phases. Vertical dotted lines are used as a reference showing the phases corresponding to the single-layer case and the end of Néel phase re-entrace in $J_{2}-J_{\perp}$ plane. II) Phase diagram of the single layer case corresponding to Ref. 9 .

where : $\mathbf{O}$ : indicates the normal ordering of the operator $\mathbf{O}$. One of the advantages of this rotational invariant decomposition is that it enables to treat ferromagnetism and antiferromagnetism on equal footing. This decomposition has been successfully used to describe quantum disordered phases in two-dimensional frustrated antiferromagnets $7-9,37,39,41,43,44$.

In order to generate a mean field theory, we perform the Hartree-Fock decoupling

$$
\begin{aligned}
\left(\overrightarrow{\mathbf{S}}_{\alpha}(\vec{x}) \cdot \overrightarrow{\mathbf{S}}_{\beta}(\vec{y})\right)_{M F} & =\left[B_{\alpha \beta}^{*}(\vec{x}-\vec{y}) \mathbf{B}_{\alpha \beta}(\vec{x}, \vec{y})\right. \\
& \left.-A_{\alpha \beta}^{*}(\vec{x}-\vec{y}) \mathbf{A}_{\alpha \beta}(\vec{x}, \vec{y})\right] \\
& -\left\langle\left(\overrightarrow{\mathbf{S}}_{\alpha}(\vec{x}) \cdot \overrightarrow{\mathbf{S}}_{\beta}(\vec{y})\right)_{M F}\right\rangle
\end{aligned}
$$

where the mean field parameters are given by

$$
\begin{aligned}
& A_{\alpha \beta}^{*}(\vec{x}-\vec{y})=\left\langle\mathbf{A}_{\alpha \beta}^{\dagger}(\vec{x}, \vec{y})\right\rangle, \\
& B_{\alpha \beta}^{*}(\vec{x}-\vec{y})=\left\langle\mathbf{B}_{\alpha \beta}^{\dagger}(\vec{x}, \vec{y})\right\rangle,
\end{aligned}
$$

and the exchange at the mean field level is

$$
\left\langle\left(\overrightarrow{\mathbf{S}}_{\alpha}(\vec{x}) \cdot \overrightarrow{\mathbf{S}}_{\beta}(\vec{y})\right)_{M F}\right\rangle=\left|B_{\alpha \beta}(\vec{x}-\vec{y})\right|^{2}-\left|A_{\alpha \beta}(\vec{x}-\vec{y})\right|^{2} .
$$

The mean field equations (6) and (7) must be solved in a self-consistent way together with the following constraint for the number of bosons in the system

$$
B_{\alpha \alpha}(\vec{R}=\overrightarrow{0})=4 N_{c} S,
$$




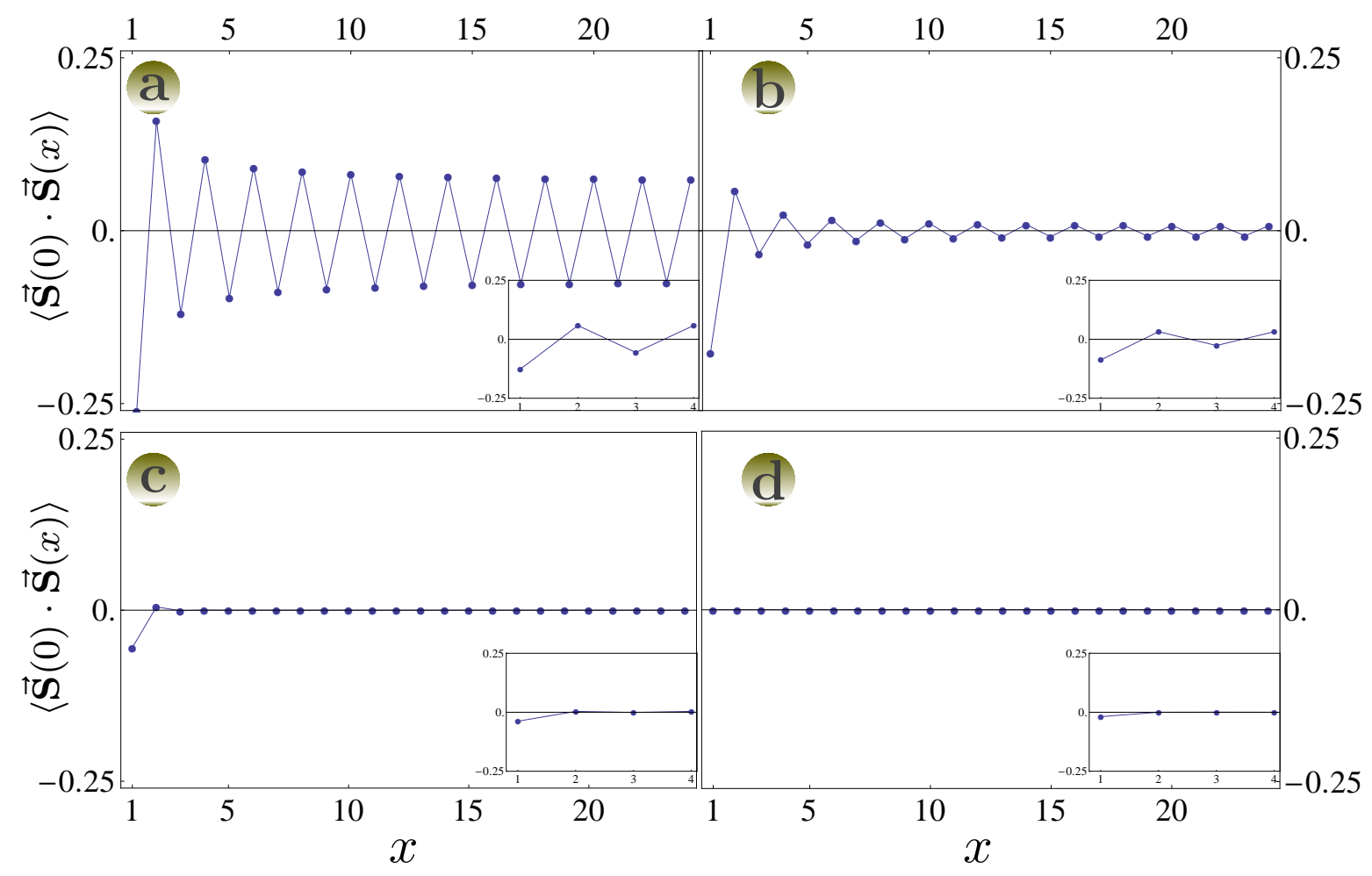

FIG. 4. (Color online) Spin-spin correlation between spins belonging to the same layer in the zig-zag direction obtained by SBMFT for a 10000 sites system. The labels a,b,c,d correspond to the points showed in Fig. $3\left(J_{\perp}=0.5,2.5,3.5,5\right)$. In the insets we show the same correlations obtained by Lanczos diagonalization of a 24 sites system.

where $N_{c}$ is the total number of unit cells and $S$ is the spin strength. We use a self consistent procedure to find mean field solutions that distinguish Néel phase from magnetically disordered phases, and in particular possible phases without translational and rotational symmetry breaking. Following the lines of Ref. 9 , we work with two sites per unit cell, which is the smallest unit cell compatible with these kind of solutions.

Self consistent solutions in the bilayer honeycomb lattice involve finding the roots of coupled nonlinear equations for the mean field parameters and solving the constraints to determine the values of the Lagrange multipliers $\lambda^{(\alpha)}$ which fix the number of bosons in the system. We perform the calculations for large systems and extrapolate the results to the thermodynamic limit. Details of the self consistent calculation can be consulted in the bibliography 7.9 .

Using SBMFT we study some features of the phase diagram in the $J_{2}-J_{\perp}$ plane. The line $J_{\perp}=0$ corresponds to the phase diagram for the single layer honeycomb lattice. A description of the phases presented in the single layer phase diagram was obtained recently using the same rotational invariant mean-field decoupling 9 . One of the advantages of the SBMFT is that allows to study large systems and perform the extrapolation to the thermodynamic limit. In particular, this is useful to determine whether the system remains gapless or not. To obtain the phase boundary between the magnetically ordered and disordered phases we use the extrapolation of the gap in the boson spectrum. In the gapless region the excitation spectrum is zero at $\vec{k}=\overrightarrow{0}$, where the boson condensation occurs, this is characteristic of the Néel ordered phase. On the other hand, in the gapped region, the absence of Bose condensation indicates that the ground state is magnetically disordered. In Figure 2 the extrapolation of the spin gap corresponding to $J_{2}=0.1$ (dashed line in Fig. (3) is presented as a function of the interlayer coupling $J_{\perp}$. For small values of the interlayer coupling the system remains gapless. As we increase $J_{\perp}$ the gap opens at a given value $J_{\perp}^{*}\left(J_{2}\right)$. Increasing more the interlayer coupling, the gap becomes a linear function of $J_{\perp}$. At the value $J_{\perp}^{*}\left(J_{2}\right)$ the Néel order is destroyed leading to the IVBC ground state. As is known, mean-field techniques are not the most convenient methods to study the properties of a system near a phase transition, so it may be difficult to determine quantitatively the transition between Néel and disordered phases using only SBMFT. For this reason, in our case, we would tend to conclude that the abrupt change of behavior in the gap $\Delta$ at $J_{\perp}=4$, Fig. 2 does not indicate a phase transition, but could be an indication of the breakdown of the mean field calculation. Actually physical quantities, as magnetization and correlations, calculated in green and light-blue shadowed regions of Fig. 2 do not show qualitative differences.

In Fig. 3 we show the phase diagram in the $J_{2}-J_{\perp}$ plane corresponding to $S=1 / 2$. For $J_{\perp} \gg J_{2}$ one can 
expect a IVBC ground state adiabatically connected with the limit of decoupled dimers, i.e. two singlets per unit cell, between spins 1(2) and 3(4) (see Fig. 1). In this limit the ground state energy per dimer is $E_{I V B C}=-\frac{3}{4} J_{\perp}$, with an energy gap $\Delta=J_{\perp}$ to triplet magnetic excitations.

In order to support the analytical results of the meanfield approach, we have also performed Lanczos ED calculations on finite systems with 24 spins and periodic boundary conditions for $S=1 / 2$. The bilayer structure of the lattice makes particularly difficult to study small systems because there are four sites per unit cell. In particular, correlation functions between spins belonging to the same layer can be studied only for a few neighbors. Fig. 4 shows the spin-spin correlation between spins belonging to the same layer in the zig-zag direction obtained by SBMFT corresponding to the points (a), (b), (c) and (d) of Fig. 3 for a 10000 sites system. The insets correspond to the results obtained for the same points with Lanczos technique on a 24 sites system. Although correlations are calculated only for a few sites with Lanczos, the absence of antiferromagnetic order in the insets of Figures 4.c and 4.d is clear. This is consistent with the SBMFT results corresponding to the main Figures 4.c and 4.d.

In Fig. 5 we show the energy per dimer in units of $J_{\perp}$ calculated with SBMFT (blue circles) and Lanczos for a system with 24 sites (red squares) for $J_{2}=0.18$. As it can be observed the energy per dimer gets very close to the value corresponding to a dimer product state $\frac{E}{2 N J_{\perp}}=-3 / 4$, as $J_{\perp}$ is increased.

As showed in Fig. 3 in the region $0.2075 \lesssim J_{2} \lesssim 0.289$ there is a reentrant effect. In this range, Néel phase separates from $J_{2}$ axis, leaving a tiny space for a magnetically disordered phase. In this way, Néel phase is here not only limited by some value $J_{\perp}^{*}\left(J_{2}\right)$ from above, but also by a second value $J_{\perp}^{* *}\left(J_{2}\right)$ (See Figure 3) from below. In Fig. 2, we show the sublattice magnetization ${ }^{9.37,38}$ along the line $J_{2}=0.1$. It is clear that a small bilayer coupling enhances the antiferromagnetic long range order, which is the reason of the reentrant effect.

On the other hand, in the range $0.3732 \lesssim J_{2} \lesssim 0.398$ $\left(J_{\perp}=0\right)$, there is evidence of the existence of an on-layer nematic VBC phase ${ }^{9}$ (see Figure $\left.3 \mathrm{HI}\right)$. In this VBC phase $\mathrm{SU}(2)$ spin rotational and lattice translational symmetries are preserved. But $Z_{3}$ symmetry, corresponding to $2 \pi / 3$ rotations around an axis perpendicular to the plane and passing through a site, is broken. By increasing the interlayer coupling $J_{\perp}$ the system moves to the IVBC where the $Z_{3}$ symmetry is recovered. In order to observe this symmetry restoring we introduce the $Z_{3}$ directional symmetry-breaking order parameter $\rho \underline{10,29}$

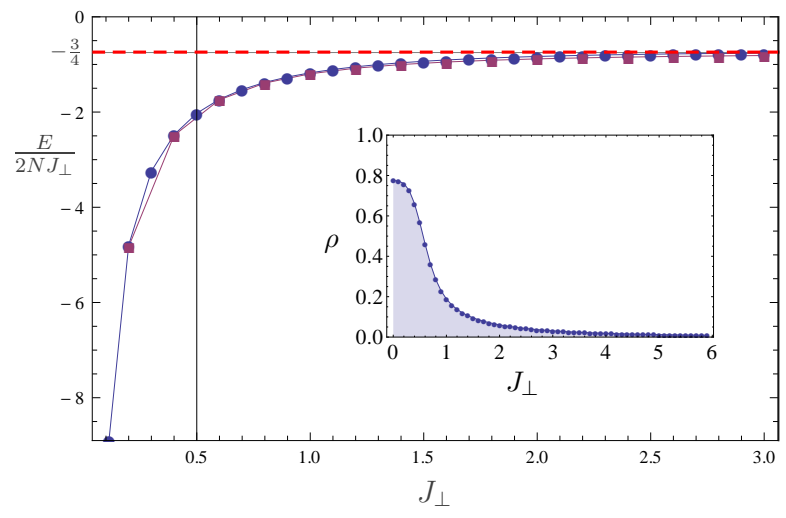

FIG. 5. (Color online) Ground state energy per dimer (in units of $J_{\perp}$ ) as a function of $J_{\perp}$ for $J_{2}=0.18$ obtained by means of SBMFT extrapolated to the thermodynamic limit (blue circles) and Lanczos for a 24 sites system (red squares). Horizontal dashed red line indicates to $\frac{E}{2 N J_{\perp}}=-3 / 4$ corresponding to the decoupled dimer product state. Inset: $Z_{3}$ directional symmetry-breaking order parameter $\rho$ as a function of $J_{\perp}$ corresponding to the line $J_{2}=0.38$.

$$
\begin{aligned}
\rho & =\frac{4}{3} \mid\left(\left\langle\overrightarrow{\mathbf{S}}_{1}(\vec{r}) \cdot \overrightarrow{\mathbf{S}}_{2}(\vec{r})\right\rangle+e^{i 2 \pi / 3}\left\langle\overrightarrow{\mathbf{S}}_{1}(\vec{r}) \cdot \overrightarrow{\mathbf{S}}_{2}\left(\vec{r}+\vec{e}_{1}\right)\right\rangle\right. \\
& \left.+e^{i 4 \pi / 3}\left\langle\overrightarrow{\mathbf{S}}_{1}(\vec{r}) \cdot \overrightarrow{\mathbf{S}}_{2}\left(\vec{r}-\vec{e}_{2}\right)\right\rangle\right) \mid
\end{aligned}
$$

This order parameter is zero when the bond energies $\kappa_{i}=\left\langle\overrightarrow{\mathbf{S}}_{1}(\vec{r}) \cdot \overrightarrow{\mathbf{S}}_{2}\left(\vec{r}+\vec{e}_{i}\right)\right\rangle$ are equal, being $i=0,1,2$ where $\vec{e}_{0}=\overrightarrow{0}, \vec{e}_{1}=\frac{1}{2}(\sqrt{3}, 3)$ and $\vec{e}_{2}=\frac{1}{2}(\sqrt{3},-3)$. The parameter is chosen to be $\rho=1$ when only one of the bond energies is nonzero. In the case $J_{\perp}=0$, this parameter is nonzero in the region $0.3732 \lesssim J_{2} \lesssim 0.398$. For small interlayer coupling, the bond energies satisfy $\kappa_{i} \neq \kappa_{j}=\kappa_{k}$. Therefore, the system is still in the nematic VBC phase. But increasing further the interlayer coupling the order parameter tends to zero continuously as shown in the inset of Figure 5 and for large $J_{\perp}$ the system enters in the IVBC. Finally, in the region $0.289 \lesssim J_{2} / J_{1} \lesssim 0.3732$ the ground state preserves $\mathrm{SU}(2)$, lattice translational and $Z_{3}$ symmetries and the spin-spin correlations are short ranged. This agrees with the evidence of a spin liquid phase in the phase diagram corresponding to $J_{\perp}=0^{9,18,19}$.

\section{LINEAR SPIN WAVE THEORY}

In this section we use a linear spin wave approach to study the stability of Néel order as a function of the spin strength. The classical spin state corresponding to the energy minimum of the Hamiltonian (11) for $J_{2}<\frac{1}{6} J_{1}$ is given by an anti-parallel (Néel) configuration. Incorporating quantum fluctuation to the classical ground state is likely to lead to the melting of Néel order. 


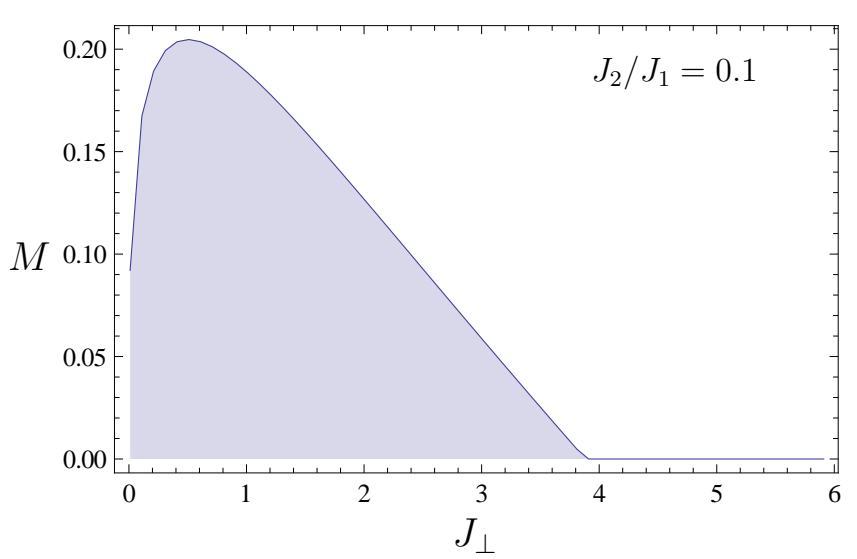

FIG. 6. (Color online) Staggered magnetization vs. $J_{\perp}$ obtained by means of linear spin wave approximation. For small values of the intralayer coupling the Néel order is enhanced, in agreement with SBMFT results.

For the spin wave implementation it is convenient to define new spin operators by rotating in $\pi$ the spins belonging to sublattices 2 and 3 (See Fig. 1) about the $x$-axis. After the rotation we have

$$
\begin{aligned}
& \tilde{S}_{\alpha}^{x}(\vec{r})=S_{\alpha}^{x}(\vec{r}) \\
& \tilde{S}_{\alpha}^{y}(\vec{r})=-S_{\alpha}^{y}(\vec{r}) \\
& \tilde{S}_{\alpha}^{z}(\vec{r})=-S_{\alpha}^{z}(\vec{r}),
\end{aligned}
$$

for spin operators belonging to sublattices 2 or 3, while $\tilde{\vec{S}}_{\alpha}(\vec{r})=\vec{S}_{\alpha}(\vec{r})$ for sublattices 1 and 4 . Thereby, the classical ground state have all spins pointing towards the new $S^{z}$ axis.

In order to study spin-wave fluctuations, we write the spin operators in terms of Holstein-Primakoff bosons as follows

$$
\begin{aligned}
\tilde{S}_{\alpha}^{+}(\vec{r}) & =\sqrt{2 S} a_{\alpha}(\vec{r}) \\
\tilde{S}_{\alpha}^{-}(\vec{r}) & =\sqrt{2 S} a_{\alpha}^{\dagger}(\vec{r}) \\
\tilde{S}_{\alpha}^{z}(\vec{r}) & =S-n_{\alpha}(\vec{r}) .
\end{aligned}
$$

The Hamiltonian can be written in terms of these boson operators as

$$
H=E_{0}+H_{S W},
$$

with

$$
\begin{aligned}
E_{0} & =2 N S^{2}\left(6 J_{2}-3 J_{1}-J_{\perp}\right) \\
H_{S W} & =2 N S\left(6 J_{2}-3 J_{1}-J_{\perp}\right) \\
& +\int d^{2} \vec{k} \overrightarrow{\mathbf{a}}^{\dagger}(\vec{k}) \mathcal{M}(\vec{k}) \overrightarrow{\mathbf{a}}(\vec{k}),
\end{aligned}
$$

where $\overrightarrow{\mathbf{a}}(\vec{k})$ is a vector of bosonic operators.

$$
\mathcal{M}(\vec{k})=\left(\begin{array}{cc}
\Gamma & \Omega \\
\Omega & \Gamma
\end{array}\right)
$$

with

$$
\begin{gathered}
\Gamma=\left(\begin{array}{cccc}
\gamma_{2}(\vec{k}) & 0 & 0 & 0 \\
0 & \gamma_{2}(\vec{k}) & 0 & 0 \\
0 & 0 & \gamma_{2}(\vec{k}) & 0 \\
0 & 0 & 0 & \gamma_{2}(\vec{k})
\end{array}\right) \\
\Omega=\left(\begin{array}{cccc}
0 & \gamma_{1}(-\vec{k}) & \gamma_{\perp} & 0 \\
\gamma_{1}(\vec{k}) & 0 & 0 & \gamma_{\perp} \\
\gamma_{\perp} & 0 & 0 & \gamma_{1}(-\vec{k}) \\
0 & \gamma_{\perp} & \gamma_{1}(\vec{k}) & 0
\end{array}\right)
\end{gathered}
$$

being functions $\gamma_{1}, \gamma_{2}$ and $\gamma_{\perp}$ given by

$$
\begin{aligned}
\gamma_{1}(\vec{k}) & =\frac{1}{2} J_{1} S\left(1+e^{i \vec{k} \cdot \vec{e}_{1}}+e^{-i \vec{k} \cdot \vec{e}_{2}}\right) \\
\gamma_{2}(\vec{k}) & =J_{2} S\left(\cos \left(\vec{k} \cdot \vec{e}_{1}\right)+\cos \left(\vec{k} \cdot \vec{e}_{2}\right)+\cos \left(\vec{k} \cdot\left(\vec{e}_{1}+\vec{e}_{2}\right)\right)\right) \\
& +\frac{3}{2} J_{1} S-3 J_{2} S+\frac{1}{2} J_{\perp} S \\
\gamma_{\perp} & =\frac{1}{2} J_{\perp} S .
\end{aligned}
$$

Then we use a Bogoliubov transformation to diagonalize the Hamiltonian $H_{S W}$ and obtain the following eigenvalues

$$
\varepsilon_{\beta}^{ \pm}(\vec{k})=\sqrt{\left(\gamma_{2}(\vec{k})\right)^{2}-\left(\gamma_{\perp} \pm\left|\gamma_{1}(\vec{k})\right|\right)^{2}},
$$

where $\beta=1,2$ is the layer index. The staggered magnetization can be calculated in the linear approximation as follows

$$
\begin{aligned}
M & =\frac{1}{4 N} \sum_{\vec{r}, \alpha} \tilde{S}_{\alpha}^{z}(\vec{r}) \\
& =S-\frac{1}{4 N} \sum_{\vec{r}, \alpha} \mathbf{a}_{\alpha}^{\dagger}(\vec{r}) \mathbf{a}_{\alpha}(\vec{r}) .
\end{aligned}
$$

On the one hand, fluctuations around the Néel state increase with the frustration $J_{2}$, and can destroy the Néel order. On the other hand, as the value of $\mathrm{S}$ is lowered, quantum fluctuations become more important and we can expect the Néel state to melt for a given value of $\mathrm{S}$. The correction to the classical boundary for the Néel state can be estimated by finding the frustration $J_{2}$ at which the sublattice magnetization $M$ given by Eq. (27) vanishes. In Fig. 6 we present the sublattice magnetization $M$ as a function of the interlayer coupling $\left(J_{\perp}\right)$ corresponding to $J_{2}=0.1$ (dashed line on Figure 3). Notice that, for small values of the interlayer coupling, the magnetization is an increasing function of $J_{\perp}$, i.e, the antiferromagnetic order is enhanced. But increasing more the value of $J_{\perp}$ the sublattice magnetization is reduced and vanishes for large values of $J_{\perp}$. This behavior is in agreement with the SBMFT results and the reentrant effect observed in Fig. 3. In Fig. 7, we present the melting curves in the $1 / S-J_{2}$ plane for different values of $J_{\perp}$. 


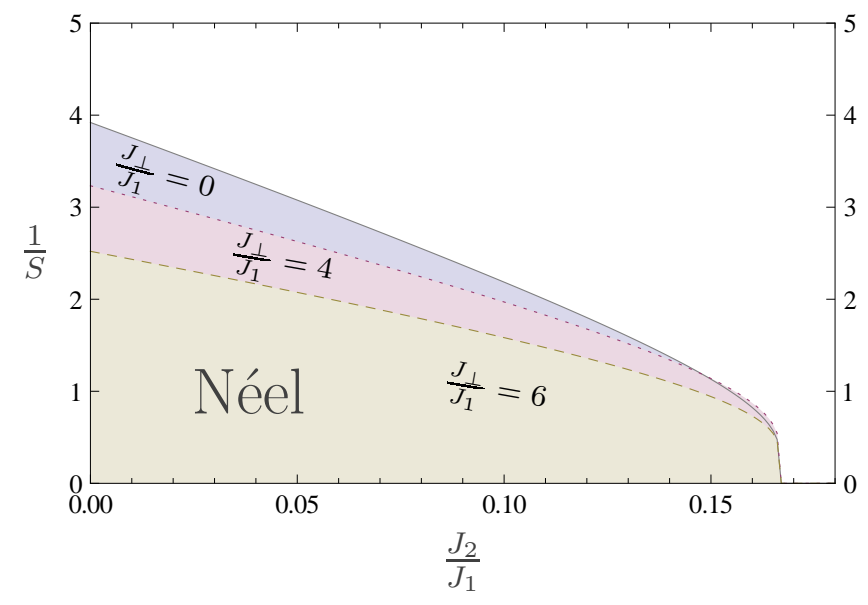

FIG. 7. (Color online) Phase diagram in the $1 / S-J_{2}$ plane for different values of $J_{\perp}$ obtained by means of LSWT.

The case corresponding to $J_{\perp}=0$ agrees with LSWT results presented in Ref. 48 (Fig. 5) for the single layer $J_{1}-J_{2}$ Heisenberg model. For large values of $J_{\perp}$ the stability region for the Néel state is reduced. Notice that in References 7 and 48 there is a discrepancy between LSWT and Schwinger bosons mean field determinations of the Néel state boundary. This difference could be reduced by means of higher order $1 / S$ corrections to the LSWT, which are beyond the scope of the present work. Finally, in Fig. 8 we show the dispersion of magnon modes along the path depicted in the inset. It is clear that as we increase the interlayer coupling, two of the four magnon modes acquires a nonzero gap at the $\Gamma$ point.

\section{COMPARISON BETWEEN SERIES EXPANSION AND EXACT DIAGONALIZATION}

As a complement to our analysis, we have performed series expansion (SE) calculations, starting from the limit of isolated dimers connecting spins from both layers via $J_{\perp}$. This allows us to assess Lanczos results by comparison with other non-mean-field technique. To this end, we have decomposed the Hamiltonian Eq.(1) into $H=H_{0}\left(J_{\perp}\right)+V\left(J_{1}, J_{2}\right)$, where $H_{0}\left(J_{\perp}\right)$ represents decoupled dimers and $V\left(J_{1}, J_{2}\right)$ is the part of Hamiltonian that connects dimers by means of $J_{1}, J_{2}$ couplings.

Since each dimer has two energy levels (singlet and triplet), the spectrum of $H_{0}\left(J_{\perp}\right)$ is equidistant, allowing to sort the levels structure of $H_{0}$ in a block-diagonal form, where each block is labeled by an energy quantumnumber Q. Ground state (vacuum) is in $\mathrm{Q}=0$ sector, i.e., all dimers are in the singlet state. $\mathrm{Q}=1$ sector is composed by states obtained by creating (from vacuum state) one-elementary triplet excitation (particle) on a given dimer, whereas that $Q \geq 2$ is of multiparticle nature.

Perturbation $V\left(J_{1}, J_{2}\right)$ does not conserve the block- diagonal form of $H_{0}\left(J_{\perp}\right)$, i.e., it mixes different Q-sectors. However, for this type of Hamiltonian, it can be shown $\underline{49}$ that it is possible to recover the block-diagonal form by means of continuous unitary transformations, using the flow equation method of Wegner ${ }^{50}$. It essentially consists in transforming $H$ onto an effective Hamiltonian $H_{\text {eff }}$ which is block-diagonal in the quantum number $Q$. This transformation can be achieved order by order in a perturbative series in powers of $J_{1,2}$, leading to $H_{\text {eff }}=H_{0}\left(J_{\perp}\right)+\sum_{n, 0<m<n} J_{1}^{n-m} J_{2}^{m} C_{n, m}$, where $C_{n, m}$ are weighted products of Q-conserving terms in $V\left(J_{1}, J_{2}\right)$, determined by recursive differential equations, see Ref. 49 for details.

$Q$-number conservation allows the evaluation of several observables directly from $H_{\text {eff }}$ in terms of a $\mathrm{SE}$ in $J_{1,2}$. For the present model we have performed $O(5)$ and $O(4) \mathrm{SE}$ in $J_{1,2}$ for ground state energy $(Q=0)$ and for triplet dispersion $(Q=1)$, respectively. Explicit expressions are too long to be printed explicitly, in particular triplet dispersion. Upon request they will be made available electronically. Regarding technical details about the calculation we refer to Ref. 51 .

To illustrate the type of results obtained, in Fig. 9 we show the ground state energy per site as a function of $J_{1}$ and $J_{2}=0$, obtained by $\mathrm{O}(5) \mathrm{SE}$ (blue circles) and $\mathrm{ED}$ on a finite system of 24 sites (red squares). As it can be observed, both techniques predict an energy decreasing with the coupling of interlayer-dimers via $J_{1}$. Furthermore, there is an excellent quantitative agreement between both methods, up to $J_{1} \simeq 0.25$. Beyond this value, the difference between the two approaches becomes increasingly noticeable, being attributable to finite size effects of ED and the order achieved in the SE. When the frustration $J_{2}$ is incorporated, the agreement is not as good as in the unfrustrated case. This might be due to the stronger effect that the frustration induces on finite size effects in Lanczos calculation.

On the other hand, triplet gap is shown in the inset of Fig. 9 for the same set of parameters as the ground state energy. Here we also observe that both techniques predict a tendency to a closure of the gap, when $J_{1}$ is turned on. While in this case we have achieved a $O(4)$ $\mathrm{SE}$, we see that the range of agreement between ED and $\mathrm{SE}$ is practically the same as before, being as well reduced when the frustration is included. Overall, our calculations shows that ED and SE share a range parameters where both predict the same behavior. A more detailed analysis in search of transitions, involving gap closure or level-crossings from SE point of view, is beyond the scope of present work.

\section{DISCUSSION AND CONCLUSIONS}

We have studied the phase diagram corresponding to a frustrated Heisenberg model on the bilayer honeycomb 

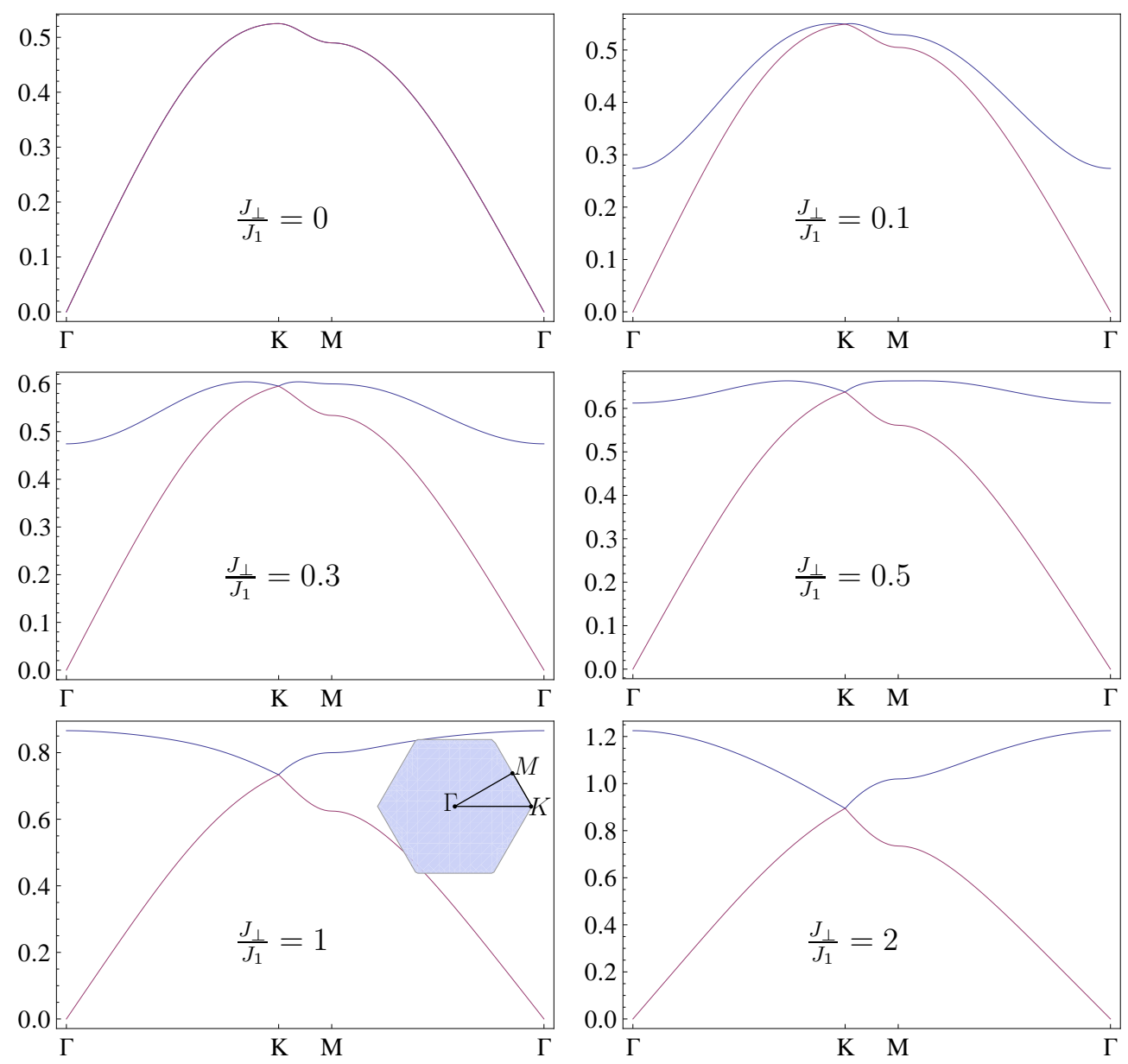

FIG. 8. (Color online) Dispersion of magnon modes corresponding to $J_{2} / J_{1}=0.1$ along the path depicted in the inset, obtained by means of LSWT.

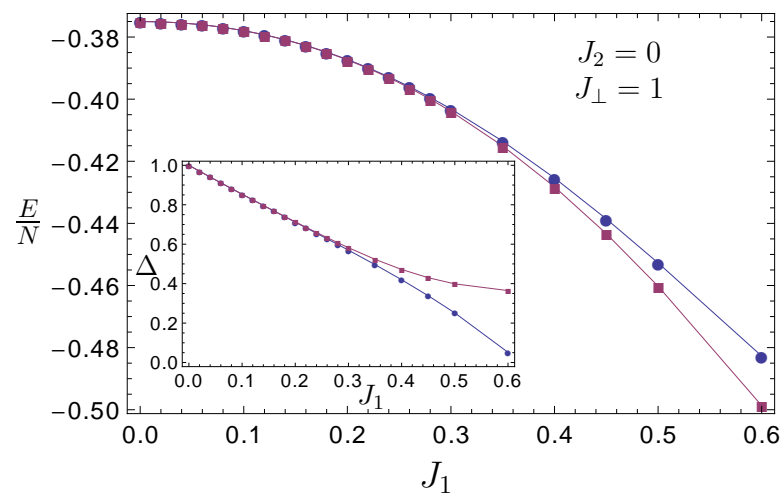

FIG. 9. (Color online) Ground state energy per site as a function of $J_{1}$ obtained by Lanczos (ED) on a 24 sites system (red squares) and $O(5)$ Series Expansion (SE) (blue circles). Inset: triplet gap (same set of parameters as main panel) ED (red squares) and $O(4) \mathrm{SE}$ (blue circles).

lattice, by means of Schwinger bosons mean field theory, complemented with exact diagonalization, linear spinwave theory and series expansion.
By analyzing the sublattice magnetization and the spin gap by SBMFT, we have described the behavior of the quantum phases as the interlayer coupling is increased. The absence of Néel order for large values of the interlayer coupling has also been observed by spin-spin correlation calculations, where SBMFT and ED techniques predict the same qualitative behavior.

In particular, in the small frustration region $\left(J_{2} / J_{1} \lesssim\right.$ $0.2075)$ the system is Néel ordered for $J_{\perp}=0$, but increasing the interlayer coupling up to a value $\left(J_{\perp}^{*}\right)$ the Néel order is destroyed and the system enters in a non magnetic phase. The spin-spin correlations are consistent with the destruction of the Néel order, given place, for large values of the interlayer coupling, to a phase with short range spin-spin correlations and a finite spin gap.

In the region $\left(0.2075 \lesssim J_{2} / J_{1} \lesssim 0.289\right)$, the phase diagram shows signatures of a reentrant behavior. At $J_{\perp}=0$ the system does not present magnetic order, but increasing the interlayer coupling up to a finite (and small) value $J^{* *}\left(J_{2}\right)$, the system becomes Néel ordered. Increasing even more the interlayer coupling, the Néel order is destroyed at a given value $J^{*}\left(J_{2}\right)$. The behavior of the sublattice magnetization as the function of $J_{\perp}$ also 
support the existence of the reentrant behavior.

For values of the interlayer coupling between $(0.289 \lesssim$ $\left.J_{2} / J_{1} \lesssim 0.3732\right)$ the Néel order is absent at $J_{\perp}=0$ and the system presents a nonzero spin gap, whereas in the region $\left(0.3732 \lesssim J_{2} / J_{1} \lesssim 0.398\right)$ each layer presents a nematic disordered phase $\mathrm{e}^{\frac{9}{}}$. In both cases increasing the value of $J_{\perp}$ the system goes to an interlayer valence bond crystal with a spin gap that is proportional to the interlayer coupling.

In all the range of values $0<J_{2} / J_{1}<0.398$, for $J_{\perp} / J_{1}>4$ the system presents signatures of an interlayer-valence bond crystal (IVBC) phase that evolves adiabatically from the limit of decoupled interlayer-dimers. This is corroborated by series expansion calculations starting explicitly from the limit of isolated interlayer dimers.

The precise determination of transitions lines between different quantum phases present in the model is not a simple task. Among these issues, the important question about how are the nematic VBC and IVBC phases connected still remains open. The mean field character of SBMFT method does not allow us to draw a definite conclusion about the real nature of the transition.
From the viewpoint of series expansion (SE), it is possible to analyze the adiabatic evolution of the nematic VBC phase, starting appropriately from isolated dimers on each plane. Thus, the possibility and type of transitions between nematic VBC and IVBC phases could be estimated by analyzing level crossings and gap closures between this SE and the dimer SE obtained in Section $\mathrm{V}$ for IVBC phase. While this analysis goes beyond the scope of this work, it clearly deserves more investigation. We postpone the detailed study of these transitions for future work, as we have focused on the general characteristics of each region of the phase diagram.

\section{ACKNOWLEDGMENTS}

We are especially grateful to Hirokazu Tsunetsugu for fruitful discussions. H.Zhang is supported by the Japanese Government Scholarship from MEXT of Japan. C. A. Lamas and M. Arlego are partially supported by CONICET (PIP 1691) and ANPCyT (PICT 1426).
* zhanghao@issp.u-tokyo.ac.jp

† lamas@fisica.unlp.edu.ar

1 P. W. Anderson, Mater. Res. Bull. 8, 153 (1973); P. Fazekas and P. W. Anderson, Phil. Mag. 30, 423 (1974); P. W. Anderson, Science 235, 1196 (1987).

2 L. Balents, Nature 464, 199 (2010).

${ }^{3}$ G. Misguich and C. Lhuillier, in Frustrated Spin Systems, edited by H. T. Diep, (World Scientific, Singapore, 2005).

4 C. A. Lamas, A. Ralko, M. Oshikawa, D. Poilblanc, and P. Pujol,Phys. Rev. B 87, 104512 (2013)

${ }^{5}$ C. Lhuillier and G. Misguich, in Introduction to Frustrated Magnetism, Eds. C. Lacroix, P. Mendels, and F. Mila, (Springer-Verlag, Berlin Heidelberg, 2011).

6 F. Wang, Phys. Rev. B 82, 024419 (2010).

7 D. C. Cabra, C. A. Lamas, and H. D. Rosales, Phys. Rev. B 83, 094506 (2011).

8 D. C. Cabra, C. A. Lamas, and H. D. Rosales, Modern Physics Letters B (MPLB) 25, 891 (2011).

9 Hao Zhang and C. A. Lamas, Phys. Rev. B 87, 024415 (2013).

10 A. Mulder, R. Ganesh, L. Capriotti and A. Paramekanti, Phys. Rev. B 81, 214419 (2010).

11 H. Mosadeq, F. Shahbazi, and S. A. Jafari, J. Phys.: Condens. Matter 23, 226006 (2011).

12 A. F. Albuquerque, D. Schwandt, B. Hetényi, S. Capponi, M. Mambrini, and A. M. Läuchli, Phys. Rev. B 84, 024406 (2011).

13 J. Reuther, D. A. Abanin, and R. Thomale, Phys. Rev. B 84, 014417 (2011).

14 D. J. J. Farnell, R. F. Bishop, P. H. Y. Li, J. Richter, and C. E. Campbell, Phys. Rev. B 84, 012403 (2011).

15 R. F. Bishop, P. H. Y. Li, D. J. J. Farnell, and C. E. Campbell, J. Phys.: Condens. Matter 24, 236002 (2012).
16 P. H. Y. Li, R. F. Bishop, D. J. J. Farnell, and C. E. Campbell, Phys. Rev. B 86, 144404 (2012).

17 R. F. Bishop, P. H. Y. Li, and C. E. Campbell, J. Phys.: Condens. Matter 25, 306002 (2013).

18 B.K. Clark, D.A. Abanin, and S.L. Sondhi Phys. Rev. Lett. 107, 087204 (2011).

19 F. Mezzacapo and M. Boninsegni, Phys. Rev. B 85, 060402(R) (2012).

20 R. Ganesh, J. van den Brink, and S. Nishimoto, Phys. Rev. Lett. 110, 127203 (2013).

21 Z. Zhu, D. A. Huse, and S. R. White, Phys. Rev. Lett. 110, 127205 (2013).

22 S. S. Gong, D. N. Sheng, O. I. Motrunich, and M. P. A. Fisher, Phys. Rev. B 88, 165138 (2013).

23 Hong-Yu Yang, A F. Albuquerque, S. Capponi, A. M Lauchli and K. P. Schmidt, New J. Phys. 14115027 (2012).

24 O. Smirnova, M. Azuma, N. Kumada, Y. Kusano, M. Matsuda, Y. Shimakawa, T. Takei, Y. Yonesaki, and N. Kinomura, J. Am. Chem. Soc., 131, 8313 (2009).

25 S. Okubo, F. Elmasry, W. Zhang, M. Fujisawa, T. Sakurai, H. Ohta, M. Azuma, O. A. Sumirnova, and N. Kumada, J. Phys.: Conf. Series 200, 022042 (2010).

26 M. Matsuda, M. Azuma, M. Tokunaga, Y. Shimakawa and N. Kumada Phys. Rev. Lett. 105, 187201 (2010).

27 M. Azuma, M. Matsuda, N. Onishi, S. Olga, Y. Kusano, M. Tokunaga, Y. Shimakawa, and N. Kumada, J. Phys.: Conf. Series 320, 012005 (2011).

28 H. C. Kandpal and J. van den Brink, Phys. Rev. B 83, 140412(R) (2011).

29 S. Okumura, H. Kawamura, T. Okubo, and Y. Motome, J. Phys. Soc. Jpn. 79, 114705 (2010).

30 R. Ganesh, D.N. Sheng, Y.-J. Kim and A. Paramekanti, Phys. Rev. B 83, 144414 (2011). 
31 R. Ganesh, S.V. Isakov, and A. Paramekanti, Phys. Rev. B 84, 214412 (2011).

32 J. Oitmaa and R. R. P. Singh, Phys. Rev. B 85, 014428 (2012).

33 E. Rastelli, A. Tassi, and L. Reatto, Physica B 97, 1 (1979).

34 J. B. Fouet, P. Sindzingre, and C. Lhuillier, Eur. Phys. J. B 20, 241 (2001).

35 T. Einarsson and H. Johannesson, Phys. Rev. B 43, 5867 (1991).

36 J. Oitmaa and R. R. P. Singh, Phys. Rev. B 84, 094424 (2011).

37 H. A. Ceccatto, C. J. Gazza, and A. E. Trumper, Phys. Rev. B 47, 12329 (1993).

38 C. J. Gazza and H. A. Ceccato, J. Phys.: Condens. Matter 5, L135 (1993).

39 A. E. Trumper, L. O. Manuel, C. J. Gazza, and H. A. Ceccatto, Phys. Rev. Lett. 78, 2216 (1997).

40 R. Flint and P. Coleman, Phys. Rev. B 79, 014424 (2009).
41 A. Mezio, C. N. Sposetti, L. O. Manuel, and A. E. Trumper, Europhys. Lett. 94, 47001 (2011).

${ }^{42}$ H. Feldner, D. C. Cabra, and G. L. Rossini, Phys. Rev. B 84, 214406 (2011).

43 L. Messio, B. Bernu, and C. Lhuillier, Phys. Rev. Lett. 108, 207204 (2012).

${ }^{44}$ L. Messio, C. Lhuillier, and G. Misguich, Phys. Rev. B 87, 125127 (2013).

45 D. P. Arovas and A. Auerbach, Phys. Rev. B 38, 316 (1988); A. Auerbach and D. P. Arovas, Phys. Rev. Lett. 61, 617 (1988).

46 A. Auerbach, Interacting Electrons And Quantum Magnetism (Springer-Verlag, New York, 1994).

47 A. Auerbach and D. P. Arovas, in Introduction to Frustrated Magnetism, Eds. C. Lacroix, P. Mendels, and F. Mila, (Springer-Verlag, Berlin Heidelberg, 2011).

48 A. Mattsson, P. Frojdh, and T. Einarsson, Phys. Rev. B 49, 3997 (1994).

49 C. Knetter and G.S. Uhrig, Eur. Phys. J. B 13, 209 (2000).

50 F. Wegner, Ann. Phys. 506, 77 (1994).

51 M. Arlego and W. Brenig, Phys. Rev. B 84, 134426 (2011). 